\title{
Dynamics of orientational fluctuations in a homeotropic Fréedericksz transition under a rotating magnetic field
}

\author{
F. Sagués \\ Departament de Química Física, Universitat de Barcelona, Diagonal 647, Barcelona 08028, Spain \\ (Received 1 September 1987; revised manuscript received 9 February 1988)
}

\begin{abstract}
We study the problem of the Fréedericksz transition under a rotating magnetic field by using a dynamical model which incorporates thermal fluctuations into the whole set of nematodynamic equations. In contrast to other geometries, nonuniform textures in the plane of the sample do not appear favored. The proper consideration of thermal noise enables us to describe the dynamics of orientational fluctuations both below and above the shifted instability.
\end{abstract}

\section{INTRODUCTION}

Liquid crystals have become an interesting subject of theoretical and practical research in the field of nonequilibrium transitions. Very broadly studied is the field-induced distortion of a nematic film with positive diamagnetic anisotropy $\chi_{a}$, called the Fréedericksz transition. ${ }^{1}$ In the context of this instability two different questions have deserved a good deal of attention in the past: (i) the time evolution of a periodically forced system, and (ii) the possibility of observing nonuniform distortions of characteristic periodicity in the plane perpendicular to the initial alignment of the sample. The Freedericksz transition under a rotating field has already been studied by Gasparoux and Prost ${ }^{2}$ in a planar-anchoring situation. Brochard and co-workers ${ }^{3,4}$ discussed this problem for a homeotropic geometry, which is the one adopted here, assuming uniformity in the plane of the slab. Much more recently, ${ }^{5}$ Kuzma dealt with a somewhat different geometry for a lyotropic material with $\chi_{a}<0$. With respect to the second question, much work has been devoted to the study of transient periodic textures of hydrodynamic nature accompanying the pure Freedericksz reorientation of the sample. Perpendicular, ${ }^{6-10}$ oblique, ${ }^{11}$ and two-dimensional ${ }^{15}$ striped patterns have been investigated theoretically and observed experimentally.

In this paper we will discuss simultaneously both aspects for a homeotropic sample placed under a rotating field perpendicular to the initial orientation of the molecules of the material. The entire set of nematodynamic equations is considered in order to retain hydrodynamic effects responsible for eventual backflow patterns. In addition, much effort is devoted to incorporating thermal fluctuations into our dynamical scheme from the beginning, to account for their essential role in triggering the relaxation from the initial unstable undistorted state and its decay to the final stable distorted configuration. This is accomplished by using a model we recently proposed in Refs. 9 and 10 generalizing previous versions without hydrodynamic coupling. ${ }^{12}$

Our results seem to preclude the appearance of regular backflow inhomogeneities regardless of the frequency and intensity of the magnetic forcing. At the same time, by including thermal noise in a consistent way a genuine periodic behavior of the fluctuations below the instability is found, whereas they amplify in a modulated way above the critical magnetic intensity.

\section{LINEARIZED EQUATIONS WITH HYDRODYNAMIC COUPLING}

Let us call $z$ the direction perpendicular to the plates containing the sample and separated by a distance $d$. A rotating magnetic field of characteristic frequency $\omega$ is applied perpendicular to the initial alignment of the directors, let us say in the plane $x-y$. If the intensity of the magnetic forcing exceeds an $\omega$-dependent threshold, to be determined later, the sample will undergo an internal homeotropic-planar reorientation. We focus on the components of the director $\left(n_{x}, n_{y}\right)$ which become macroscopically amplified during the realignment. To take into account hydrodynamic and thermal noise effects we use a Langevin version of the nematodynamic equations as established in Refs. 9 and 10. Specializing this scheme to the geometry here considered and invoking a minimal-coupling approximation, 9,10 the final equations for the director and velocity components read (summation over repeated indices generically denoted $\alpha$ is implied) as shown in Eq. (1) (see next page). In (1) $\rho$ is the mass density, $\gamma_{1}, \gamma_{2}\left(\lambda=-\gamma_{2} / \gamma_{1}\right), v_{1}, v_{2}$, and $v_{3}$ are the viscosity coefficients used in Harvard's version of the nematodynamic equations, $p$ is the pressure, and $\mathcal{F}$ stands for the free energy whose contributions reduce in the limit of small distortions to

$$
\begin{aligned}
\frac{\delta \mathcal{F}}{\delta n_{x}}= & -K_{1}\left(\partial_{x}^{2} n_{x}+\partial_{x y}^{2} n_{y}\right)+K_{2}\left(\partial_{x y}^{2} n_{y}-\partial_{y}^{2} n_{x}\right) \\
& -K_{3} \partial_{z}^{2} n_{x}-\chi_{a}\left(n_{x} H_{x}+n_{y} H_{y}\right) H_{x} \\
\frac{\delta \mathcal{F}}{\delta n_{y}}= & -K_{1}\left(\partial_{x y}^{2} n_{x}+\partial_{y}^{2} n_{y}\right)+K_{2}\left(\partial_{x y}^{2} n_{x}-\partial_{x}^{2} n_{y}\right) \\
& -K_{3} \partial_{z}^{2} n_{y}-\chi_{a}\left(n_{x} H_{x}+n_{y} H_{y}\right) H_{y} \\
\frac{\delta \mathcal{F}}{\delta v_{\alpha}}= & \rho v_{\alpha}, \quad \alpha=\{x, y, z\} .
\end{aligned}
$$



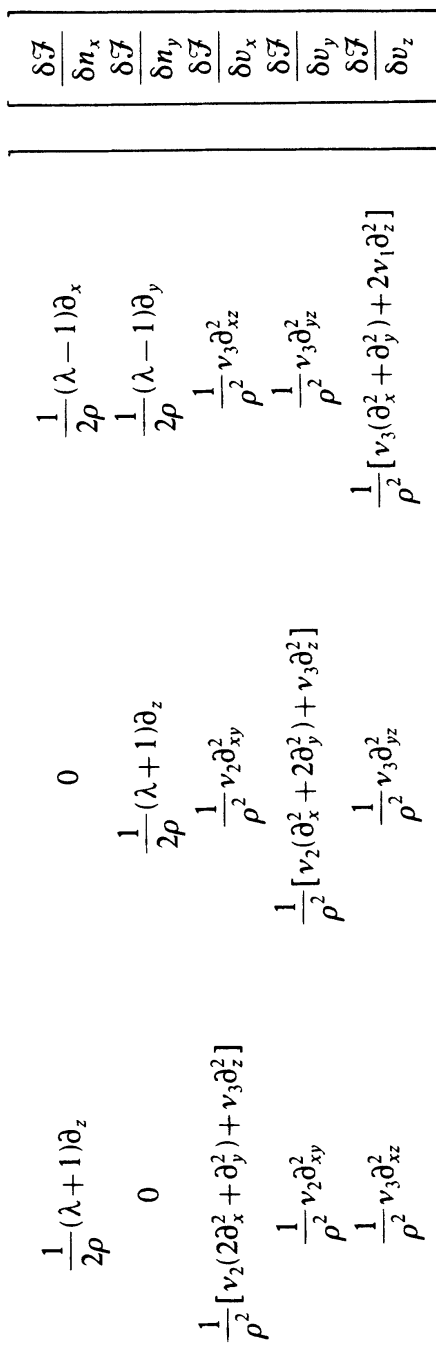

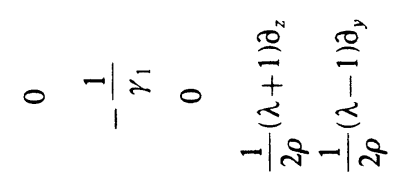

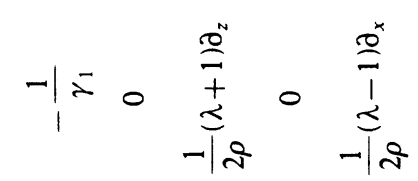

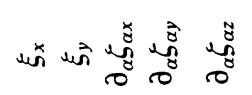$$
00 \frac{0}{2} 0^{2} \frac{0}{2}
$$$$
-10
$$

$\|$

$$
2^{x} \quad 2^{2} a^{2} a^{2}
$$

The Gaussian random forces left in (1) have zero mean value and satisfy appropriate fluctuation-dissipation relations

$\left\langle\xi_{\alpha}\left(\mathbf{r}_{1}, t_{1}^{\prime}\right) \xi_{\beta}\left(\mathbf{r}_{2}, t_{2}^{\prime}\right)\right\rangle=2 \delta_{\alpha \beta} \frac{k_{B} T}{\gamma_{1}} \delta\left(\mathbf{r}_{1}-\mathbf{r}_{2}\right) \delta\left(t_{1}^{\prime}-t_{2}^{\prime}\right)$,

$$
\left\langle\zeta_{\alpha \beta}\left(\mathbf{r}_{1}, t_{1}^{\prime}\right) \zeta_{\delta \gamma}\left(\mathbf{r}_{2}, t_{2}^{\prime}\right)\right\rangle=2 k_{B} T M_{\alpha \beta \delta \gamma} \delta\left(\mathbf{r}_{1}-\mathbf{r}_{2}\right) \delta\left(t_{1}^{\prime}-t_{2}^{\prime}\right),
$$

with $M_{\alpha \beta \delta \gamma}$ expressed in terms of the initial components of the director

$$
\begin{aligned}
M_{\alpha \beta \delta \gamma}=\frac{1}{\rho^{2}}[ & 2\left(v_{1}+v_{2}-2 v_{3}\right) n_{\alpha}^{0} n_{\beta}^{0} n_{\delta}^{0} n_{\gamma}^{0} \\
& +v_{2}\left(\delta_{\alpha \delta} \delta_{\beta \gamma}+\delta_{\alpha \gamma} \delta_{\beta \delta}\right) \\
& +\left(v_{3}-v_{2}\right)\left(n_{\alpha}^{0} n_{\delta}^{0} \delta_{\beta \gamma}+n_{\alpha}^{0} n_{\gamma}^{0} \delta_{\beta \delta}+n_{\beta}^{0} n_{\delta}^{0} \delta_{\alpha \gamma}\right. \\
& \left.\left.+n_{\beta}^{0} n_{\gamma}^{0} \delta_{\alpha \delta}\right)\right] .
\end{aligned}
$$

To proceed further we make the common approximation of negligible inertia, we conveniently manipulate the set of equations for the velocities to get rid of pressure terms, and furthermore we invoke incompressibility of the material to eliminate one of the components of the velocity. All this algebra, leading finally to a pair of equations for the director components, is much more easily handled by Fourier transforming according to

$$
\begin{aligned}
& n_{\alpha}=\sum_{\mathbf{q}, k_{z}} n_{\alpha ; \mathrm{q}, k_{z}}\left(t^{\prime}\right) e^{i \mathbf{q} \cdot \rho \cos k_{z} z}, \\
& \xi_{\alpha}=\sum_{\mathbf{q}, k_{z}} \xi_{\alpha ; \mathbf{q}, k_{z}}\left(t^{\prime}\right) e^{i \mathbf{q} \cdot \rho} \cos k_{z} z, \\
& v_{\alpha}=\sum_{\mathbf{q}, k_{z}} v_{\alpha ; \mathbf{q}, k_{z}}\left(t^{\prime}\right) e^{i \mathbf{q} \cdot \rho \sin k_{z} z}, \\
& \left(\begin{array}{c}
\zeta_{\alpha \beta} \\
\zeta_{z z}
\end{array}\right)=\sum_{\mathbf{q}, k_{z}}\left(\begin{array}{l}
\zeta_{\alpha \beta ; \mathbf{q}, k_{z}}\left(t^{\prime}\right) \\
\zeta_{z z ; \mathbf{q}, k_{z}}\left(t^{\prime}\right)
\end{array}\right) e^{i \mathbf{q} \cdot \rho \sin k_{z} z}, \\
& v_{z}=\sum_{\mathbf{q}, k_{z}} v_{z ; \mathbf{q}, k_{z}}\left(t^{\prime}\right) e^{i \mathbf{q} \cdot \rho} \cos k_{z} z, \\
& \zeta_{\alpha z}=\sum_{\mathbf{q}, k_{z}} \zeta_{\alpha z ; \mathbf{q}, k_{z}}\left(t^{\prime}\right) e^{i \mathbf{q} \cdot \rho} \cos k_{z} z, \\
& k_{z}=(2 m+1) \frac{\pi}{d}, \quad\{\alpha, \beta\}=\{x, y\},
\end{aligned}
$$

where strong-anchoring assumptions for the director and free-free boundary conditions for the velocities are prescribed at the limiting plates: $z= \pm d / 2$. Using an opportune matrix notation for the Fourier amplitudes, the final equations read

$\gamma_{\alpha \beta} \frac{d}{d t^{\prime}} n_{\rho}\left(t^{\prime}\right)=K_{\alpha \beta} n_{\beta}+\gamma_{\alpha \beta} \xi_{\beta}+\psi_{\alpha}, \quad\{\alpha, \beta\}=\{x, y\}$.

Before giving explicit expressions for the viscosities we recall the definitions of Miesowicz's coefficients 


$$
\begin{aligned}
& \eta_{a} \equiv v_{2} \\
& \eta_{b} \equiv \nu_{3}+\frac{1}{4} \gamma_{1}(1-\lambda)^{2}, \\
& \eta_{c} \equiv v_{3}+\frac{1}{4} \gamma_{1}(1+\lambda)^{2},
\end{aligned}
$$

we introduce a coupling viscosity

$$
\eta_{4} \equiv \frac{1}{2}\left(\eta_{b}+\eta_{c}-\gamma_{1}\right)
$$

and we define a set of auxiliary quantities

$$
\begin{aligned}
r_{1}^{2} \equiv & \eta_{c} k_{z}^{2}+\eta_{a}\left(q_{x}^{2}+q_{y}^{2}\right) \\
r_{2}^{2} \equiv & \left(\eta_{a}-2 \eta_{4}+2 v_{1}\right) k_{z}^{2}+\eta_{b}\left(q_{x}^{2}+q_{y}^{2}\right) \\
r^{4} \equiv & \eta_{c} k_{z}^{4}+2\left(\eta_{a}-\eta_{4}+v_{1}\right) k_{z}^{2}\left(q_{x}^{2}+q_{y}^{2}\right) \\
& +\eta_{b}\left(q_{x}^{2}+q_{y}^{2}\right)^{2}
\end{aligned}
$$

to finally obtain

$$
\begin{aligned}
& \gamma_{x x}=\gamma_{1}\left[1-\frac{\gamma_{1}}{4}(1+\lambda)^{2} \frac{k_{z}^{2}}{r_{1}^{2} r^{4}}\left(r_{1}^{2} k_{z}^{2}+r_{2}^{2} q_{y}^{2}\right)-\frac{\gamma_{1}}{4}\left(1-\lambda^{2}\right) \frac{q_{x}^{2}}{r^{4}}\left(\frac{1-\lambda}{1+\lambda}\left(q_{x}^{2}+q_{y}^{2}\right)+2 k_{z}^{2}\right)\right], \\
& \gamma_{y y}=\gamma_{1}\left[1-\frac{\gamma_{1}}{4}(1+\lambda)^{2} \frac{k_{z}^{2}}{r_{1}^{2} r^{4}}\left(r_{1}^{2} k_{z}^{2}+r_{2}^{2} q_{x}^{2}\right)-\frac{\gamma_{1}}{4}\left(1-\lambda^{2}\right) \frac{q_{y}^{2}}{r^{4}}\left(\frac{1-\lambda}{1+\lambda}\left(q_{x}^{2}+q_{y}^{2}\right)+2 k_{z}^{2}\right)\right], \\
& \gamma_{x y}=\gamma_{y x}=\gamma_{1}\left[\frac{\gamma_{1}}{4}(1+\lambda)^{2} \frac{k_{z}^{2}}{r_{1}^{2} r^{4}} r_{2}^{2} q_{x} q_{y}-\frac{\gamma_{1}}{4}\left(1-\lambda^{2}\right) \frac{q_{x} q_{y}}{r^{4}}\left(\frac{1-\lambda}{1+\lambda}\left(q_{x}^{2}+q_{y}^{2}\right)+2 k_{z}^{2}\right)\right] .
\end{aligned}
$$

The contributions from the elastic and magnetic torques read

$$
\begin{aligned}
& K_{x x}=\chi_{a} H^{2} \cos ^{2} \omega t^{\prime}-\left(K_{1} q_{x}^{2}+K_{2} q_{y}^{2}+K_{3} k_{z}^{2}\right), \\
& K_{y y}=\chi_{a} H^{2} \sin ^{2} \omega t^{\prime}-\left(K_{1} q_{y}^{2}+K_{2} q_{x}^{2}+K_{3} k_{z}^{2}\right), \\
& K_{x y}=K_{y x}=\chi_{a} H^{2} \sin \omega t^{\prime} \cos \omega t^{\prime}-\left(K_{1}-K_{2}\right) q_{x} q_{y} .
\end{aligned}
$$

Finally, two well-differentiated noise contributions appear in (6). $\xi_{\alpha}$ is directly related to the dynamics of the distortion modes, whereas $\psi_{\alpha}$ enters into that dynamics through the equations for the velocity fields. The explicit expressions for $\psi_{x}$ and $\psi_{y}$ in terms of $\zeta_{\alpha \beta}$ are rather complicated. We first define

$$
\begin{aligned}
\zeta_{1} \equiv \rho\left[i \zeta_{x y}\left(q_{x}^{2}-q_{y}^{2}\right)+i\left(\zeta_{y y}-\zeta_{x x}\right) q_{x} q_{y}\right. \\
\left.-k_{z}\left(q_{x} \zeta_{y z}-q_{y} \zeta_{x z}\right)\right], \\
\zeta_{2} \equiv \rho\left[-i k_{z}\left(q_{x} \zeta_{x x}+q_{y} \zeta_{x y}\right)-\left(q_{x}^{2}-k_{z}^{2}\right) \zeta_{x z}\right. \\
\left.\quad-q_{x} q_{y} \zeta_{y z}+i q_{x} k_{z} \zeta_{z z}\right],
\end{aligned}
$$

to get finally

$$
\begin{aligned}
& \psi_{x}=-\frac{\gamma_{1}}{2}(1+\lambda) \frac{1}{r_{1}^{2} r^{4}}[ {\left[k_{z}+\frac{1-\lambda}{1+\lambda} \frac{q_{x}^{2}}{k_{z}}\right]\left(q_{y} r_{2}^{2} \zeta_{1}+k_{z} r_{1}^{2} \zeta_{2}\right) } \\
&\left.-\frac{1-\lambda}{1+\lambda} \frac{q_{y}}{k_{z}}\left\{\left[\eta_{c} k_{z}^{4}+2\left(\eta_{a}-\eta_{4}+v_{1}\right) q_{x}^{2} k_{z}^{2}+\eta_{a} q_{y}^{2} k_{z}^{2}+\eta_{b} q_{x}^{2}\left(q_{x}^{2}+q_{y}^{2}\right)\right] \zeta_{1}-r_{1}^{2} q_{y} k_{z} \zeta_{2}\right\}\right], \\
& \psi_{y}=-\frac{\gamma_{1}}{2}(1+\lambda) \frac{1}{r_{1}^{2} r^{4}}\left[\frac{1-\lambda}{1+\lambda} \frac{q_{x} q_{y}}{k_{z}}\left(q_{y} r_{2}^{2} \zeta_{1}+k_{z} r_{1}^{2} \zeta_{2}\right)\right. \\
&\left.+\left[\frac{k_{z}}{q_{x}}+\frac{1-\lambda}{1+\lambda} \frac{q_{y}^{2}}{q_{x} k_{z}}\right]\left\{r_{1}^{2} q_{y} k_{z} \zeta_{2}-\left[\eta_{c} k_{z}^{4}+2\left(\eta_{a}-\eta_{4}+v_{1}\right) q_{x}^{2} k_{z}^{2}+\eta_{a} q_{y}^{2} k_{z}^{2}+\eta_{b} q_{x}^{2}\left(q_{x}^{2}+q_{y}^{2}\right)\right] \zeta_{1}\right\}\right] .
\end{aligned}
$$

A series of manipulations turn out to be particularily useful in what follows. First of all we assume we are dealing with small enough magnetic fields so that only the first $z$ mode, $k_{z}=\pi / d$, will become unstable as a result of the Fréedericksz transition. This enables us to utilize throughout the remainder of the paper dimensionless wave numbers $\mathbf{Q}=\mathbf{q} / \pi / d$. Expressions (9)-(11) transform then into

$$
\begin{aligned}
& R_{1}^{2} \equiv \eta_{c}+\eta_{a}\left(Q_{x}^{2}+Q_{y}^{2}\right) \\
& R_{2}^{2} \equiv\left(\eta_{a}-2 \eta_{4}+2 v_{1}\right)+\eta_{b}\left(Q_{x}^{2}+Q_{y}^{2}\right), \\
& R^{4} \equiv \eta_{c}+2\left(\eta_{a}-\eta_{4}+v_{1}\right)\left(Q_{x}^{2}+Q_{y}^{2}\right)+\eta_{b}\left(Q_{x}^{2}+Q_{y}^{2}\right)^{2},
\end{aligned}
$$




$$
\begin{aligned}
& \gamma_{x x}=\gamma_{1}\left[1-\frac{\gamma_{1}}{4}(1+\lambda)^{2} \frac{1}{R_{1}^{2} R^{4}}\left(R_{1}^{2}+R_{2}^{2} Q_{y}^{2}\right)-\frac{\gamma_{1}}{4}\left(1-\lambda^{2}\right) \frac{Q_{x}^{2}}{R^{4}}\left(\frac{1-\lambda}{1+\lambda}\left(Q_{x}^{2}+Q_{y}^{2}\right)+2\right)\right], \\
& \gamma_{y y}=\gamma_{1}\left[1-\frac{\gamma_{1}}{4}(1+\lambda)^{2} \frac{1}{R_{1}^{2} R^{4}}\left(R_{1}^{2}+R_{2}^{2} Q_{x}^{2}\right)-\frac{\gamma_{1}}{4}\left(1-\lambda^{2}\right) \frac{Q_{y}^{2}}{R^{4}}\left(\frac{1-\lambda}{1+\lambda}\left(Q_{x}^{2}+Q_{y}^{2}\right)+2\right)\right], \\
& \gamma_{x y}=\gamma_{y x}=\gamma_{1}\left[(1+\lambda) \frac{R_{2}^{2}}{R_{1}^{2}}-(1-\lambda)\left(\frac{1-\lambda}{1+\lambda}\left(Q_{x}^{2}+Q_{y}^{2}\right)+2\right] \mid \frac{\gamma_{1}}{4}(1+\lambda) \frac{1}{R^{4}} Q_{x} Q_{y},\right. \\
& K_{x x}=\chi_{a} H^{2} \cos ^{2} \omega t^{\prime}-\pi^{2} / d^{2}\left(K_{1} Q_{x}^{2}+K_{2} Q_{y}^{2}+K_{3}\right), \\
& K_{y y}=\chi_{a} H^{2} \sin ^{2} \omega t^{\prime}-\pi^{2} / d^{2}\left(K_{1} Q_{y}^{2}+K_{2} Q_{x}^{2}+K_{3}\right), \\
& K_{x y}=K_{y x}=\chi_{a} H^{2} \sin \omega t^{\prime} \cos \omega t^{\prime}-\pi^{2} / d^{2}\left(K_{1}-K_{2}\right) Q_{x} Q_{y} .
\end{aligned}
$$

Secondly we propose a time adimensionalization and a notation in terms of reduced magnetic fields

$t \equiv t^{\prime} \frac{1}{\gamma_{1}} K_{3} \frac{\pi^{2}}{d^{2}}, \quad h^{2} \equiv H^{2} / H_{c}^{2}, \quad H_{c}^{2}=\frac{1}{\chi_{a}} K_{3} \frac{\pi^{2}}{d^{2}}$,

which converts (6) into

$$
\Gamma_{\alpha \beta} \frac{d}{d t} n_{\beta}(t)=K_{\alpha \beta}^{r} n_{\beta}+\Psi_{\beta},
$$

with $\Gamma_{\alpha \beta}=\gamma_{1}^{-1} \gamma_{\alpha \beta}$, and the elements of the reduced matrix $K^{r}$ being

$$
\begin{aligned}
& K_{x x}^{r}=h^{2} \cos ^{2} \Omega t-\left(\frac{K_{1}}{K_{3}} Q_{x}^{2}+\frac{K_{2}}{K_{3}} Q_{y}^{2}+1\right), \\
& K_{y y}^{r}=h^{2} \sin ^{2} \Omega t-\left(\frac{K_{1}}{K_{3}} Q_{y}^{2}+\frac{K_{2}}{K_{3}} Q_{x}^{2}+1\right), \\
& K_{x y}^{r}=K_{y k}^{r}=h^{2} \sin \Omega t \cos \Omega t-\frac{1}{K_{3}}\left(K_{1}-K_{2}\right) Q_{x} Q_{y}, \\
& \Omega \equiv \omega \frac{\gamma_{1}}{K_{3} \pi^{2} / d^{2}} .
\end{aligned}
$$

Finally, $\Psi$ collects the entire contribution arising from thermal fluctuations

$$
\Psi_{\alpha} \equiv \frac{1}{K_{3} \frac{\pi^{2}}{d^{2}}}\left(\gamma_{1} \Gamma_{\alpha \beta} \xi_{\beta}+\psi_{\alpha}\right)
$$

\section{MEAN-FIELD DYNAMICS}

The first important point we want to address in this paper concerns the possibility of finding nonuniform distortions $\mathbf{Q} \neq 0$, leading the response of the system to the Fréedericksz instability. This can be discussed from the explicit expressions obtained for the viscosities (15) and elastic and magnetic torques (19). As expected, we can directly conclude from (19) that the nonuniform rotation modes involve additional contributions corresponding to internal elastic distortions which are absent for the uniform rotation mode $\mathbf{Q}=\mathbf{0}$. Owing to that, the only possibility for finding nonuniform patterns in the $x-y$ plane developing faster than the pure uniform one should be justified in a tradeoff of rotation for shear viscosities if their effective values for modes $\mathbf{Q} \neq \mathbf{0}$ were sensibly smaller than for $\mathbf{Q}=\mathbf{0}$. Actually, this is the usual argument employed in explaining the common striped structures resulting from the Fréedericksz instability in the pure twist $^{7,9,10}$ and planar-homeotropic ${ }^{6,11,13}$ geometries in nematic materials with $\chi_{a}>0$. However, in our case here, and using typical values for the material parameters corresponding to $N$-(4- $n$-methoxy)benzylidene- $4^{\prime}-(n-$ butyl)aniline (MBBA) at room temperatures, ${ }^{1}$ the effective viscosities do not fulfill this requirement. According to (15) all the viscosity components increase in going from a uniform mode $\mathbf{Q}=0$ to nonuniform ones $\mathbf{Q} \neq \mathbf{0}$ of moderate modulus for which we can still predict instabilities under the conditions previously prescribed of low magnetic fields. Specifically, the diagonal viscosities $\Gamma_{x x}$ and $\Gamma_{y y}$ increase from a nonzero positive value, $1-\gamma_{1}(1+\lambda)^{2} / 4 \eta_{c}$, whereas $\Gamma_{x y}$ augments from a null value at the origin of reduced wave numbers. In passing we note that this argument applies regardless of the intensity and frequency of the forcing and consequently it is also valid for the static case $\Omega=0$. Thus hydrodynamic effects cannot be invoked in the homeotropic geometry to explain domain growth, but they result in an effective viscosity even for the dynamics of the dominant reorientational mode, i.e., the homogeneous $\mathbf{Q}=0$ mode.

Once this important point has been stated clearly we will restrict our discussion to the most unstable mode $\mathbf{Q}=\mathbf{0}$. In this way we recover what could be considered as a mean-field description of the dynamics of the nematic sample. The explicit expressions for the components of $\Gamma, K^{r}$, and $\Psi$ are then greatly simplified,

$$
\begin{aligned}
& \Gamma_{x x}=\Gamma_{y y}=1-\frac{\gamma_{1}}{4} \frac{(1+\lambda)^{2}}{\eta_{c}}, \quad \Gamma_{x y}=0, \\
& K_{x x}^{r}=h^{2} \cos ^{2} \Omega t-1, \\
& K_{y y}^{r}=h^{2} \sin ^{2} \Omega t-1, \\
& K_{x y}^{r}=h^{2} \sin \Omega t \cos \Omega t, \\
& \Psi_{\alpha}= \frac{\gamma_{1}}{K_{3} \pi^{2} / d^{2}}\left[\left[1-\frac{\gamma_{1}}{4} \frac{(1+\lambda)^{2}}{\eta_{c}}\right] \xi_{\alpha}\right. \\
&\left.\quad-\frac{(1+\lambda)}{2 \eta_{c}} \rho \xi_{\alpha z}\right], \quad \alpha=\{x, y\} .
\end{aligned}
$$


It turns out to be suitable to solve (18) in terms of an azimuthal angle $\phi(t)$,

$$
\left[\begin{array}{l}
n_{x}(t) \\
n_{y}(t)
\end{array}\right)=\theta(t)\left(\begin{array}{l}
\cos \phi(t) \\
\sin \phi(t)
\end{array}\right] .
$$

In view of (22) the essential variable concerning the instability is $\theta(t)$, since $\phi(t)$ merely reproduces the rotating character of the applied magnetic field without telling us anything about the intrinsic stability properties of the initial configuration. Since we are mainly interested in studying the effect of thermal fluctuations on the dynamics of the variable really experiencing the instability, we adopt here a strategy which consists in neglecting contributions in the evolution of $\phi(t)$. Thus the equation for the azimuthal angle reads

$$
\left(1-\frac{\gamma_{1}}{4} \frac{(1+\lambda)^{2}}{\eta_{c}}\right) \frac{d}{d t} \phi(t)=\frac{h^{2}}{2} \sin 2(\Omega t-\phi),
$$

independently of $\theta(t)$. On the other hand, for the distortion angle we get

$$
\begin{aligned}
\left(1-\frac{\gamma_{1}}{4} \frac{(1+\lambda)^{2}}{\eta_{c}}\right) \frac{d}{d t} \theta(t)= & {\left[h^{2} \cos ^{2}(\Omega t-\phi)-1\right] \theta(t) } \\
& +\left(\cos \phi \Psi_{x}+\sin \phi \Psi_{y}\right) .
\end{aligned}
$$

Apart from the $\Psi$ term in (24), Eqs. (23) and (24) coincide with the ones used by Brochard et al. ${ }^{4}$ where backflow effects were disregarded from the beginning, except for the reducing factor in the viscosity: ${ }^{14} \gamma_{1}^{*} / \gamma_{1}$ $=1-\gamma_{1}(1+\lambda)^{2} / 4 \eta_{c} \simeq 0.2$. This fact alters correspondingly the analysis that follows referred to the phase diagram $\left(h^{2}, \Omega\right)$, although the analysis qualitatively reproduces the results of Ref. 4.

Two well-differentiated regimes can be distinguished regarding the rotation of the director.

(i) A synchronous rotation characterized by a constant retardation angle $\alpha$ :

$$
\begin{aligned}
& \phi(t)=\Omega t-\alpha, \\
& \sin 2 \alpha=\Omega \tau, \\
& \tau \equiv \frac{2}{h^{2}}\left(1-\frac{\gamma_{1}}{4} \frac{(1+\lambda)^{2}}{\eta_{c}}\right),
\end{aligned}
$$

this solution being acceptable provided $\Omega \tau \leq 1$. This defines a threshold for synchronous rotation

$$
h_{\mathrm{syn}}(\Omega)=\left[2 \Omega\left[1-\frac{\gamma_{1}}{4} \frac{(1+\lambda)^{2}}{\eta_{c}}\right]\right]^{1 / 2},
$$

or in the original variables

$$
H_{\mathrm{syn}}(\omega)=\left[\frac{2 \omega}{\chi_{a}} \gamma_{1}\left[1-\frac{\gamma_{1}}{4} \frac{(1+\lambda)^{2}}{\eta_{c}}\right]\right]^{1 / 2},
$$

to be compared with the result in Ref. 4: $H_{\text {syn }}(\omega)=\left(2 \omega \gamma_{1} / \chi_{a}\right)^{1 / 2}$.

(ii) an asynchronous rotation characterized by a timedependent retardation angle $\alpha(t)$ :

$$
\begin{aligned}
\phi(t)=\Omega t-\alpha(t) & , \\
\tan \alpha(t)=\frac{1}{\Omega \tau}+ & \left(1-\frac{1}{\Omega^{2} \tau^{2}}\right)^{1 / 2} \\
& \times \tan \left(\left(\Omega^{2} \tau^{2}-1\right)^{1 / 2} \frac{t}{\tau}\right),
\end{aligned}
$$

this solution applying when $\Omega \tau \geq 1$.

\section{DYNAMICS OF THE ORIENTATIONAL FLUCTUATIONS}

In this section we want to describe the behavior of orientational fluctuations following the Fréedericksz transition. We will be mainly interested in the previously identified asynchronous regime, since in this case the dynamics corresponds to a nonautonomous system.

First of all we convert (24) into an equation for the variance $C(t)=\left\langle\theta^{2}(t)\right\rangle$. This is commonly done by standard procedures ${ }^{15}$ which explicitly use the correlations of the stochastic forces given in ( 3 and 4 ). The final equation for $C(t)$ reads

$$
\begin{aligned}
\frac{d}{d t} C(t)= & \frac{2}{\left(1-\frac{\gamma_{1}}{4} \frac{(1+\lambda)^{2}}{\eta_{c}}\right]} \\
& \times\left\{\left[h^{2} \cos ^{2}(\Omega t-\phi)-1\right] C(t)+\epsilon\right\},
\end{aligned}
$$

where $\epsilon$ is a dimensionless measure of the intensity of the thermal noise ${ }^{16}(V$, volume of the sample):

$$
\epsilon=2 \frac{k_{B} T}{V K_{3} \pi^{2} / d^{2}} .
$$

The consistency of the Langevin approach used throughout this paper is immediately recognized by noting that the term responsible for thermal fluctuations appears in (28) affected by exactly the same viscosityreducing factor as the magnetoelastic one. This enables us to rescale time according to

$$
\begin{aligned}
& \frac{d}{d s} C(s)=2\left[h^{2} \cos ^{2}\left(\Omega_{s} s-\phi\right)-1\right] C(s)+2 \epsilon, \\
& s \equiv t /\left(1-\frac{\gamma_{1}}{4} \frac{(1+\lambda)^{2}}{\eta_{c}}\right], \quad \Omega_{s} \equiv \Omega \frac{t}{s} .
\end{aligned}
$$

The term $2 \epsilon$, which could be called the Cook term in close analogy with the Cahn-Hilliard-Cook theory of spinodal decomposition, is a genuine consequence of the inclusion of thermal noise from the beginning and is missed in Brochard et al.'s deterministic treatment. Its importance will be discussed later on. First we study (30) in the two limits previously identified.

(i) In the synchronous regime $\alpha$ is constant so that $C(s)$ evolves with an effective reduced field smaller by a factor $\cos ^{2} \alpha$,

$$
\cos ^{2} \alpha=\frac{1}{2}\left[1+\left(1-\Omega^{2} \tau^{2}\right)^{1 / 2}\right],
$$

with respect to the static case. The conclusion regarding the critical field is then 


$$
h_{c}^{2}\left(\Omega_{s}\right)=1+\Omega_{s}^{2},
$$

or in the original variables

$$
\frac{H_{c}^{2}(\omega)}{H_{c}^{2}}=1+\left(\frac{\gamma_{1} \omega}{K_{3} \pi^{2} / d^{2}}\right)^{2}\left(1-\frac{\gamma_{1}}{4} \frac{(1+\lambda)^{2}}{\eta_{c}}\right)^{2},
$$

which consistently reproduces the result of Ref. 4

$$
H_{c}^{2}(\omega) / H_{c}^{2}=1+\left(\gamma_{1} \omega / K_{3} \pi^{2} / d^{2}\right)^{2}
$$

except for the viscosity-reducing factor.

(ii) In the asynchronous regime we have

$$
\frac{d}{d s} C(s)=2\left(\frac{h^{2}}{1+\tan ^{2} \alpha(s)}-1\right) C(s)+2 \epsilon,
$$

with

$$
\begin{aligned}
\tan \alpha(s)=\frac{h^{2}}{2} \frac{1}{\Omega_{s}}+ & {\left[1-\left[\frac{h^{2}}{2} \frac{1}{\Omega_{s}}\right]^{2}\right]^{1 / 2} } \\
& \times \tan \left\{\left[\Omega_{s}^{2}-\left[\frac{h^{2}}{2}\right]^{2}\right]^{1 / 2} s\right\} .
\end{aligned}
$$

For $h^{2}<1$ the system is clearly stable. For $h^{2}>1$ we have solved (32) numerically with initial conditions corresponding to an initial field $h_{i}^{2}<1, C(0)=\left(1-h_{l}^{2}\right)^{-1} \epsilon$.

What follows is essentially an analysis of the solutions of the nonautonomous equation (32). Orientational fluctuations described by $C(s)$ are essential in the study of the initial growth right after the system begins to feel the instability. In the situation considered here we can expect that above the instability this growth will be periodically modulated. Fluctuations appear in (32) through the consistent initial conditions and also through the effect of thermal noise during the evolution incorporated via the Cook term $2 \epsilon$.

Two very different behaviors reproduce the long-time dynamics of $C(s)$ after transients, depending on initial conditions, have been forgotten: (a) a region of bounded periodic oscillations with period

$$
T=\pi /\left(\Omega_{s}^{2}-\left(h^{2} / 2\right)^{1 / 2},\right.
$$

and (b) a region of unbounded oscillations. This very different dynamics is shown in Fig. 1 where we plot for a particular value of $\Omega_{s}, \Omega_{s}=3$, a situation of stable oscillations for $h^{2}=1.65$, and a linearly unstable one with $h^{2}=2.1$.

Clearly these two behaviors correspond, respectively, to situations below and above the instability. We have numerically solved (32) and (33) at different points of the phase diagram $\left(h^{2}, \Omega_{s}\right)$ in order to locate the instability. Interestingly, the instability condition turns out to be independent of $\Omega_{s}$ in the asynchronous regime examined here and corresponds to $h^{2}\left(\Omega_{s}\right)=2$ in agreement with Brochard's analysis.

The very different behaviors shown in Fig. 1 may also be analyzed in terms of the somewhat different role played by thermal noise, measured by $\epsilon$ below and above the instability. Below the instability a sudden change from $h_{i}^{2}$ to $h^{2}$ with $2>h^{2}>h_{i}^{2}$ results in a controlled and limited amplification of fluctuations which finally get trapped into a "limit cycle" behavior characteristic of the stable equilibrium fluctuations appropriate to the successive and periodic determinations of the magnetic field [Fig. 1(a)]. If we let $\epsilon$ go to zero any virtual fluctuation, i.e., of nonthermal origin, has to die out, inevitably as is depicted in the inset of Fig. 1(a). On the other hand, if we increase the magnetic intensity beyond the shifted instability, $h_{i}^{2}<h^{2}, h^{2}>2$, fluctuations grow very fast, corresponding to a linearly unstable solution here modulated by the periodic forcing. A nonzero value of $\epsilon$ guarantees in this case the proper inclusion of the initial thermal fluctuations necessary to trigger the amplification phenomenon, and once it is initiated favors it especially during the early stages of the transition.

In passing we make a final comment regarding the range of validity of this linear approach. In general, this could be estimated as the lifetime of the unstable state
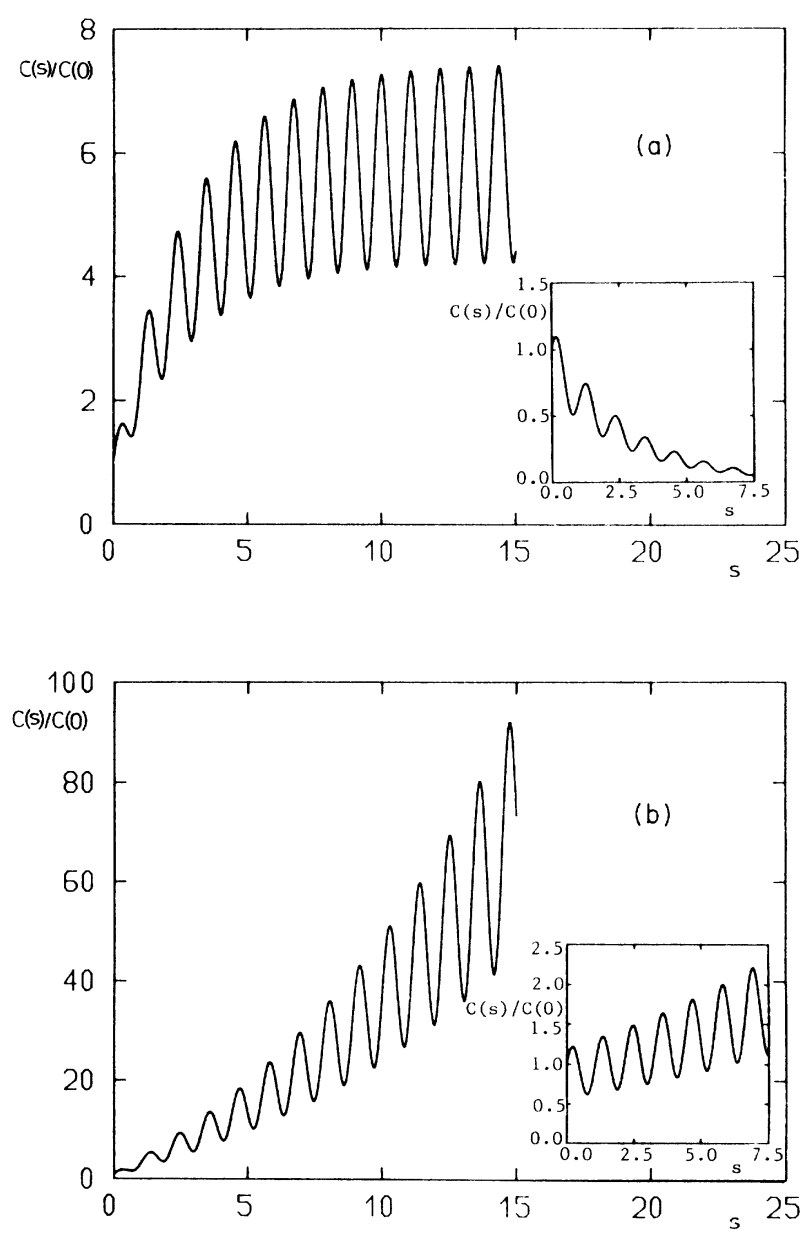

FIG. 1. Time evolution of the variance of the fluctuations corresponding to the distortion angle, $C(s)$, in the asynchronous regime (see text). Initial conditions correspond to $h_{l}^{2}=0$ whereas $\Omega_{\mathrm{s}}=3$. In (a) we show a stable evolution with $h^{2}=1.65$. In (b) a linearly unstable dynamics is depicted for $h^{2}=2.1$. In the insets we reproduce the results corresponding to Eq. (30) without the Cook contribution (see text). 
formally characterized in terms of the mean first-passage time (MFPT) to leave the vicinity of the unstable state. Although we did not attempt to evaluate it in our situation here, previous calculations referring to the standard Fréedericksz transition ${ }^{9,10,12,15}$ indicate that this time is of experimental relevance, since it is considerably larger, of the order of tenths to hundreds of seconds depending on the value of $h^{2}$, than in other experimental situations such as those corresponding to the spinodal decomposition of systems with short-range forces.

\section{CONCLUSIONS}

We have studied the onset of the Fréedericksz instability for a nematic homeotropic sample subjected to a rotating magnetic field. We have centered our analysis in the dynamical behavior of the orientational fluctuations. In the synchronous regime those fluctuations evolve autonomously according to an effective and constant magnetic intensity. In the asynchronous mode the fluctuation dynamics is nonautonomous. In this case it shows a bounded periodic behavior or a modulated pattern of continuous growth, respectively, below or above the magnetic instability. The methodological scheme used throughout the paper is based on the utilization of a Langevin version of the entire set of nematodynamic equations. This allows us to analyze hydrodynamic contributions. Effective rotational viscosities have been calculated, but no domain-structure formation phenomenon is predicted arising in backflow effects. Since this result is actually independent of the frequency of the rotating magnetic field, it also applies to the standard homeotropic Fréedericksz transition.

\section{ACKNOWLEDGMENTS}

Thanks are due to F. Arias for helping me prepare Fig. 1. Financial support from Comisón Asesora de Investigación Científica y Técnica (CAICYT), Spain, Project No. PR-84-0361, is acknowledged.
${ }^{1}$ P. G. de Gennes, The Physics of Liquid Crystals (Clarendon, Oxford, 1975).

${ }^{2}$ H. Gasparoux and J. Prost, J. Phys. (Paris) 32, 953 (1971).

${ }^{3}$ F. Brochard, J. Phys. (Paris) Lett. 35, L19 (1974).

${ }^{4}$ F. Brochard, L. Léger, and R. B. Meyer, J. Phys. (Paris) Colloq. 36, C1-209 (1975).

${ }^{5}$ M. R. Kuzma, Phys. Rev. Lett. 57, 349 (1986).

${ }^{6}$ E. Guyon, R. B. Meyer, and J. Salán, Mol. Cryst. Liq. Cryst. 54, 261 (1979).

${ }^{7}$ F. Lonberg, S. Fraden, A. J. Hurd, and R. B. Meyer, Phys. Rev. Lett. 521903 (1984).

${ }^{8}$ Y. W. Hiu, M. R. Kuzma, M. San Miguel, and M. M. Labes, J. Chem. Phys. 83, 288 (1985).

${ }^{9}$ M. San Miguel and F. Sagués, Phys. Rev. A 36, 1883 (1987).
${ }^{10}$ F. Sagués, F. Arias, and M. San Miguel, Phys. Rev. A 37, 3601 (1988).

${ }^{11}$ A. J. Hurd, S. Fraden, F. Lonberg, and R. B. Meyer, J. Phys. (Paris) 46, 905 (1985).

${ }^{12}$ F. Sagués and M. San Miguel, Phys. Rev. A 33, 2769 (1986).

${ }^{13}$ F. Sagués and F. Arias, following paper, Phys. Rev. A 38, 5367 (1988).

${ }^{14}$ F. Brochard, P. Pieranski, and E. Guyon, Phys. Rev. Lett. 28, 1681 (1972); P. Pieranski, F. Brochard, and E. Guyon, J. Phys. (Paris) 34, 35 (1973).

${ }^{15}$ F. Sagués and M. San Miguel, Phys. Rev. A 32, 1843 (1985).

${ }^{16}$ For typical samples of MBBA $\left(S=1 \mathrm{~cm}^{2}, d=10^{-2} \mathrm{~cm}\right)$ at room temperatures, $\epsilon$ can be estimated to be of order $10^{-10}$. 